\title{
Teste de dígitos no ruído baseado em software para português brasileiro em crianças com transtorno do processamento auditivo central
}

\author{
Software-based digits-in-noise test \\ for Brazilian Portuguese in children with \\ central auditory processing disorder
}

\section{Prueba de dígitos en ruido basado en software para portugués brasileño en niños con trastorno del central procesamiento auditivo}

\author{
Thalinny da Costa Silva* (D) \\ Aryelly Dayane da Silva Nunes* (D) \\ Thainá Ruth França de Farias* \\ Ana Beatriz Santos* (iD \\ Sheila Andreoli Balen* (iD
}

\section{Resumo}

Introdução: Nas situações da vida diária, frequentemente, é necessário reconhecer a fala em ambientes com ruído competitivo, sendo que dificuldades neste reconhecimento é uma queixa comum de pessoas com transtornos do processamento auditivo central (TPAC). O Teste de Dígitos no Ruído (TDR), desenvolvido para triagem de perdas auditivas em adultos e idosos, apresenta vantagens que o tornam promissor para triagem em crianças com TPAC. Objetivo: Verificar o desempenho de crianças com TPAC no TDR baseado em software para o Português brasileiro. Método: A amostra de conveniência foi constituída por 31 crianças (8-12 anos), sendo 23 com TPAC, alocadas no G1, e oito sem o transtorno, que compuseram o G2. Todas realizaram avaliação audiológica básica, timpanometria, reconhecimento numérico visual e aleatório, aplicação do TDR e avaliação comportamental do processamento auditivo central (PAC) para determinar presença ou não do TPAC. Na análise foi utilizado Teste de Mann-Whitney

* Universidade Federal do Rio Grande do Norte, Natal, Rio Grande do Norte, Brasil.

Contribuição dos autores:

TCS: concebeu e realizou a coleta e análise de dados, bem como redação do artigo científico.

ADSN: contribuiu na coleta e análise dos dados, bem como na redação do artigo científico.

TRFF e ABS: contribuiram na coleta dos dados, organização e normatização do artigo científico.

SAB: concebeu o estudo, supervisionou a coleta, realizou análise dos dados, bem como redação do artigo científico.

E-mail para correspondência: Sheila Andreoli Balen - sheila@sheilabalen.com.br Recebido: $16 / 05 / 2020$

Aprovado: 04/09/2020 
no comparativo entre G1 e G2 e correlação de Sperman entre os testes de PAC e a média da relação S/R (RSR) do TDR. Resultados: G1 e G2 apresentaram desempenhos significativamente diferentes nos testes fala filtrada (orelha direita), dicótico de dígitos (orelha esquerda), fusão binaural e gaps-in-noise de ambas as orelhas e no teste de padrões de frequência. A média da relação sinal/ruído (RSR) do TDR foi de -7,29 dB (dp $\pm 4,76)$ nos sujeitos do $\mathrm{G} 1$ e de $-8,42 \mathrm{~dB}(\mathrm{dp} \pm 2,93)$ no G2. Não houve diferença estatisticamente significante entre G1 e G2 no comparativo das médias de RSR final ( $p=0,227)$. Não foi evidenciada correlação na maior parte dos testes de PAC e o TDR, exceto correlação negativa no FBOE e DDIOD. Conclusão: O desempenho das crianças com TPAC é similar ao de crianças sem TPAC no teste de dígitos no ruído em Português Brasileiro,

Palavras-chave: Audição; Transtornos da Percepção Auditiva; Programas de Rastreamento.

\section{Abstract}

Speech recognition on noise is an auditory processing skill, important for children in contexts with competitive noise and reverberation and can test their functional capacity. The Digits-in-Noise (DIN) Test, developed for hearing screening in adults and the elderly, has advantages that make it promising for screening children with central auditory processing disorders (CAPD). Objective: Verify the performance of children with central auditory processing disorder in a software-based DIN Test for Brazilian Portuguese. Methods: The convenience sample comprised 31 children ( 8 to 12 years), 23 with CAPD placed in G1, and eight without the disorder that composed G2. All children underwent basic audiological assessment and tympanometry, visual and random numerical recognition, use of DIN and auditory processing behavioral assessment to determine the presence or absence of APD. We used the Mann-Whitney test to compare G1 and G2. Results: G1 and G2 presented different performances in the filtered speech (right ear), dichotic digits (left ear), binaural fusion and gaps-in-noise tests of both ears and in the frequency pattern test. We observed that the average DIN signal-to-noise ratio (SNR) was $-7.29 \mathrm{~dB}(\mathrm{SD} \pm 4.76)$ in $\mathrm{G} 1$ subjects, and $-8.42 \mathrm{~dB}(\mathrm{SD} \pm 2.93)$ in $\mathrm{G} 2$ subjects. There was no statistically significant difference between G1 and G2 in comparison of average final SNR $(p=0.227)$. Conclusion: Considering the average values of the final SNR, we found that the children's performance in the DIN test was similar between both groups.

Keywords: Hearing; Auditory Perceptual Disorders; Mass Screening.

\section{Resumen}

Introducción: Reconocimiento de habla en ruido es una habilidad del procesamiento auditivo, importante para chicos en contextos con ruido competitivo y reverberación, y puede ser evaluada para determinar su capacidad funcional. La Prueba de Dígitos en Ruido (PDR), desarrollada para detectar pérdida de audición en adultos y ancianos, presentado ventajas que lo hacen prometedor para detección en niños con trastorno del procesamiento auditivo central (TPAC). Objetivo: Verificar el rendimiento de niños conTPAC en PDR basado en software en portugués brasileño. Metodos: La muestra de conveniencia consistió en 31 niños entre 8-12 año, 23 chicos con TPAC, asignado en G1, y ocho sin trastorno, que componen G2. Todos realizaron evaluación audiológica básica, timpanometría, reconocimiento numérico visual y aleatorio, aplicación del PDR y evaluación comportamental del procesamiento auditivo, aplicación del PDR e del comportamiento del procesamiento auditivo para determinar la presencia o ausencia de TPAC. Se utilizó la prueba de Mann-Whitney para análisis entre G1 y G2. Resultados: G1 y G2 presentaron diferentes rendimientos en pruebas de habla filtrada (oído derecho), dígitos dicóticos (oído izquierdo), fusión binaural y gaps-in-noise en ambos oídos y en la prueba del patrón de frecuencia. La Relación Señal/Ruido (S/R) del PDR fue -7.29 dB ( $\mathrm{sd} \pm 4.76)$ en chicos del G1 y $-8.42 \mathrm{~dB}(\mathrm{sd} \pm 2.93)$ en G2. No hubo diferencias estadísticamente significativas entre G1 y G2 al comparar las medias finales de RSR ( $p=0.227)$. Conclusión: Teniendo en cuenta valores de la media $S / R$ final, hubo similitud de rendimiento en PDR en ambos grupos.

Palabras clave: Pruebas auditivas; Inteligibilidad del habla; Ruido; Pruebas de discriminación del habla; Percepción auditiva 


\section{Introdução}

Nas situações da vida diária, frequentemente, é necessário reconhecer a fala em ambientes com ruído competitivo, sendo que dificuldades neste reconhecimento é uma queixa comum de pessoas com transtornos do processamento auditivo central $(\mathrm{TPAC})^{1,2}$.

O reconhecimento de fala no ruído pode ser avaliado por meio de testes que buscam replicar situações da vida real como forma de determinar a capacidade funcional na vida cotidiana ${ }^{3}$. Isto envolve apresentar simultaneamente estímulos de fala e um ruído competitivo, como no teste de fala no ruído ${ }^{4}$, o subteste de Fala no Ruído $+8 \mathrm{~dB}$, presentes no SCAN ${ }^{5,6}$; o subteste Speech Perception in Noise (SPIN) da ferramenta Screening Test for Auditory Processing (STAP) ${ }^{7}$, o subteste Palavras no Ruído do teste Münchner Screening of Auditory Perception Disorders (MAUS) ${ }^{8}$, o Hearing in Noise Test (HINT) ${ }^{9,10}$ e o Teste de Dígitos no Ruído (TDR) baseado em software ${ }^{3,11-15}$.

A utilização de dígitos como estímulos de fala se mostra um método vantajoso por serem palavras de alta frequência e muito comuns no repertório lexical da maior parte da população, incluindo as crianças na faixa etária escolar ${ }^{12}$.

O TDR é um teste desenvolvido originalmente na Holanda ${ }^{11}$ como um teste de triagem auditiva. As pessoas ouvem trios de dígitos com ruído (espectro médio de fala) em diferentes relações sinal/ ruído nas duas orelhas simultaneamente. Para a realização do teste por telefone, os arquivos de áudio, previamente gravados por uma voz feminina, foram reproduzidos através de uma placa de som de um computador, em seguida roteados para um modem acoplado diretamente à linha telefônica. Um software era responsável por atender ao telefone e detectar as teclas pressionadas pelo ouvinte no teclado. De acordo com a resposta dada pelo ouvinte, a relação sinal-ruído era calculada para a próxima apresentação, seguindo a proposição do processo adaptativo ${ }^{11}$. Isto envolve referir que, de forma automatizada, no momento que a pessoa erra o sistema, o sinal de fala é elevado em intensidade em relação ao ruído e, à medida que acerta o sinal de fala é abaixado. Desta forma, é estabelecido para cada pessoa o limiar de reconhecimento de fala no ruído ${ }^{11}$.

O TDR foi adaptado para a língua inglesa em um aplicativo por pesquisadores da Universidade de Pretória, na África do Sul ${ }^{13}$. O estudo inicial ${ }^{13}$ evidenciou não haver diferença no uso do aplicativo com os fones de ouvido do smartphone em relação aos fones dos audiometros. Foi constatado $94 \%$ de sensibilidade e $77 \%$ de especificidade na identificação da deficiência auditiva com ponto de corte de $-9,55 \mathrm{~dB}^{14}$. Outro estudo ${ }^{15}$ evidenciou $83 \%$ de sensibilidade e $72 \%$ de especificidade com ponto de corte em $-9,8 \mathrm{~dB}$. Em ambos os estudos ${ }^{14,15} \mathrm{com}$ estímulos dióticos.

O TDR foi aplicado em crianças na faixa etária de 4 a 12 anos $^{16}$ com o intuito de avaliar o reconhecimento de fala no ruído em condições dióticas e dicóticas. Observou-se que com o aumento da idade, houve melhora significativa do limiar de reconhecimento dos dígitos no ruído. Além disso, crianças com idades entre 10 e 12 anos apresentaram desempenhos semelhantes aos do adulto ${ }^{16}$.

No Brasil, o TDR vem sendo estudado em três etapas distintas, envolvendo a tradução, adaptação e validação para o Português brasileiro. Na primeira etapa foi executado o protocolo do estudo original de Potgieter et al. ${ }^{13}$ abrangendo gravação feita por um nativo, processamento e equalização do estímulo sonoro; inserção dos dígitos em trios no software já desenvolvido; coleta de dados normativos e análise de diferentes fones de ouvido ${ }^{17}$. Esta etapa foi possível pela colaboração internacional entre três Instituições de Ensino Superior do Brasil, uma Universidade e uma empresa na Africa do Sul. $\mathrm{O}$ resultado do estudo ${ }^{17}$ desta primeira etapa foi a versão em teste do aplicativo para ser utilizado nas pesquisas no Brasil, bem como o estudo de dois diferentes tipos de fones de ouvido em adultos normo-ouvintes, sendo evidenciado que o desempenho não muda em função dos fones de ouvido ${ }^{17}$.

A segunda etapa envolveu o estudo ${ }^{18}$ da acurácia do TDR na identificação de perdas auditivas em adolescentes, adultos e idosos, evidenciado $92,73 \%$ de sensibilidade e $67,11 \%$ de especificidade do TDR nesta população, com ponte de corte de $-6,9 \mathrm{~dB}^{18}$.

A proposta no TDR é para avaliar o reconhecimento de fala no ruído, desenvolvido e estudado, predominantemente, para a triagem de perdas auditivas $^{11-15}$. No entanto, já foi estudado o seu uso no diagnóstico audiológico conjuntamente com a audiometria tonal liminar ${ }^{19}$. Sugere-se que ele possa ser uma ferramenta que auxilie no gerenciamento e aconselhamento do aparelho auditivo em pessoas com deficiência auditiva ${ }^{19}$. 
Na perspetiva de investigar outras aplicabilidades do TDR e tomando-se como referência que o reconhecimento de fala no ruído é comumente inserido em propostas de rastreio ou de triagem dos TPAC como no teste SCAN ${ }^{5,6}$ a terceira etapa dos estudos do TDR em Português Brasileira foi delineado por meio do estudo apresentando neste artigo, sendo o primeiro envolvendo crianças com e sem TPAC e o TDR em Português Brasileiro.

Assim, o objetivo deste estudo foi verificar o desempenho de crianças com TPAC no Teste Dígitos no Ruído (TDR) baseado em software para o português brasileiro.

\section{Método}

Este estudo é observacional, transversal e prospectivo. A seleção da amostra por conveniência ocorreu pelo recrutamento de crianças provenientes de escolas públicas de Natal, Rio Grande do Norte. Este estudo foi aprovado pelo Comitê de Ética em Pesquisa da Instituição, sob parecer n ${ }^{\circ} 3.232 .691$. Os pais/responsáveis das crianças participantes deste estudo assinaram o Termo de Consentimento Livre e Esclarecido (TCLE), enquanto as crianças assinaram o Termo de Assentimento Livre e Esclarecido (TALE).

Os critérios de inclusão para a pesquisa foram: (1) estar dentro da faixa etária de 8 a 12 anos; (2) apresentar respostas auditivas em até $25 \mathrm{~dB} \mathrm{NA}^{20}$ ou em intensidade menor nas frequências de 250 a $8000 \mathrm{~Hz}$; (3) timpanometria com o pico de máxima complacência na faixa de +50 a -100 da PA e com complacência estática maior ou igual a $0,2 \mathrm{cc}^{21}$; podendo ou não apresentar TPAC.

Foram excluídas crianças que não atenderam os critérios de inclusão, bem como não passaram na triagem de reconhecimento visual dos números ou não concluíram todos os procedimentos de avaliação da pesquisa.

A amostra foi dividida em dois grupos: G1 constituído por crianças que apresentaram diagnóstico de TPAC e o G2 por crianças com normalidade no PAC.

Inicialmente, foram coletadas informações para caracterização sociodemográfica da amostra, quando foi aplicado o questionário do Critério de Classificação Econômica Brasil da Associação Brasileira de Empresas de Pesquisa ${ }^{22}$ com os responsáveis pelas crianças. A partir do somatório dos itens informados por estes foi estabelecida a classi- ficação socioeconômica. A partir dos resultados foi realizado agrupamento das classificações A1, A2, B1 e B2 que se enquadraram como nível socioeconômico alto; $\mathrm{C} 1$ e $\mathrm{C} 2$ como nível socioeconômico médio; e D e E como nível socioeconômico baixo.

Os procedimentos aplicados foram: 1) teste de triagem de reconhecimento visual dos números; 2) teste de dígitos no ruído (TDR); 3 ) audiometria tonal liminar e 4) bateria de testes de avaliação do PAC. Estes procedimentos foram realizados sempre nesta ordem por pesquisadores independentes que foram mantidos cegos quanto aos resultados do TDR, da audiometria tonal liminar e da avaliação do PAC durante todo o processo avaliativo.

$\mathrm{O}$ teste de triagem de reconhecimento visual dos números foi aplicado no intuito de verificar se as crianças conseguiam reconhecer visualmente de forma correta os números que escutavam. Desta forma, foi elaborada uma prancha com algarismos de zero a nove distribuídos aleatoriamente e solicitou-se que a criança apontasse cada um deles à medida que $o$ avaliador citava, bem como realizasse a nomeação de cada um na ordem que estava disposta na prancha. $\mathrm{O}$ conhecimento e a capacidade de reconhecer visualmente os números foi uma condição para a execução da tarefa do TDR.

A versão do TDR utilizada do aplicativo, neste estudo, foi uma versão teste traduzida e adaptada para o Português Brasileiro ${ }^{17}$ instalado no smartphone da marca Motorola e modelo Z, com os fones de ouvido próprios do smartphone. Os pesquisadores instruíram as crianças e, após, realizaram o cadastro da mesma no aplicativo quando eram inseridos os fones de ouvido. Antes do início do teste, houve a apresentação de sequências de números aleatórios através do qual era possível a criança regular a intensidade inicial do estímulo baseado no seu conforto auditivo. A duração média do teste foi de aproximadamente três minutos. As crianças ouviam três dígitos (de 0 a 9 ) com ruído de fundo (ruído de fala) na intensidade de $70 \mathrm{~dB}$ NPS, na condição inphase, e deveriam reconhecer visualmente no teclado do aplicativo o número ouvido.

Após, dava-se início ao teste quando se apresentava 23 sequências de 3 dígitos cada e, ao ouvir o estímulo, a criança digitava, através do teclado que o aplicativo dispõe, quais os dígitos ouvidos. Aqueles que não puderam ser identificados deveriam ser adivinhados ${ }^{13}$. Baseado nas assertivas ou falhas, a relação sinal-ruído (S/R) entre estímulo e ruído era alterada, onde, inicialmente apresenta- 
va-se na relação $\mathrm{S} / \mathrm{R}$ de $-2 \mathrm{~dB}$ (nível mais fácil) e dificultava-se de forma automatizada caso houvesse o acerto, ou regredia de acordo com os erros, em um processo adaptativo, baseado nas seguintes relações S/R: -2, -4, -6, -8, -10, -12, -14, -16, -18 e -20 dB. Os acertos foram contabilizados quando todos os dígitos foram colocados corretamente na sequência apresentada ${ }^{13}$. Ao final, foi calculada a média das relações sinal/ruído encontrada nos acertos entre as sequências 4 e 23. Além disso, gerou-se uma tabela com os resultados de cada sequência apresentada ${ }^{18}$.

A audiometria tonal liminar por via aérea nas frequências de 250 a $8000 \mathrm{~Hz}$ foi realizada com o audiômetro da OtometricsMadsen Itera II, em cabina acústica, sendo apresentado tom puro por meio dos fones TDH 39. A timpanometria foi realizada com o aparelho Interacoustic AT235, a fim de avaliar integridade da orelha média, certificando-se da ausência de alterações condutivas.

Foram aplicados os protocolos de avaliação comportamental do PAC dentro da cabina acústica, por meio do CD de Pereira e Schochat ${ }^{4}$ acoplado ao audiômetro, composto por uma faixa com o índice percentual de reconhecimento de fala (IPRF) gravado; Teste de Fala Filtrada (FF) e o Teste de Fusão Binaural (FB) e Teste Dicótico de Dígitos (TDD) - tarefa de integração binaural. Além desses, as crianças foram avaliadas com o Teste Padrão de Frequência (TPF), na tarefa de nomeação, e o teste de resolução temporal Gaps in Noise (GIN) ${ }^{23}$. A metodologia de aplicação de cada teste e os valores normativos adotados por faixa-etária seguiram os preconizados por Pereira e Schochat ${ }^{4}$ nos testes verbais, e pela Auditec ${ }^{23}$ no TPF e GIN.

Para comprovar a presença do TPAC, as crianças deveriam apresentar, no mínimo, dois testes abaixo do esperado para a idade na avaliação do processamento auditivo central ${ }^{1}$, sendo então enquadradas ao G1. As crianças sem alterações na bateria de testes foram alocadas no G2 $2^{1,2}$. Embora tenha sido utilizado este critério para formação dos grupos foi realizada também a análise e descrição do percentual de ocorrência do desempenho alterado do G1 em cada teste e orelha, para caracterização da amostra em relação aos testes da bateria de avaliação comportamental do PAC. O mesmo não foi realizado com o $\mathrm{G} 2$, visto que este apresentou resultados normais nos testes da bateria de avaliação do PAC.
Os dados foram tabulados em formato de planilha no programa Excel e importados para o software SPSS (Statistical Package for the Social Sciences) 20.0 para posterior análise estatística.

Após, foi verificado com o teste Shapiro-Wilk que não havia distribuição normal das variáveis estudadas, sendo indicado o uso de testes não paramétricos. Desta forma, foi comparado o desempenho de cada grupo em cada teste e orelha e a comparação do desempenho no TDR entre os grupos utilizando o Teste de Mann-Whitney. Por fim, utilizou-se a correlação de Spearman para analisar se havia correlação entre cada teste da bateria de avaliação do PAC com os resultados do TDR. Adotou-se o nível de significância de 5\%.

\section{Resultados}

Foram contatadas 63 crianças, sendo que 40 compareceram para realização dos procedimentos. Na Figura 1 podem-se observar os critérios de exclusão de nove crianças, sendo a amostra composta por 31 crianças, divididas em 23 crianças no G1, com TPAC, e oito crianças no G2, sem TPAC.

Quanto ao sexo, a distribuição se deu por 13 crianças do sexo masculino no G1 (56,52\%) e sete no G2 $(87,5 \%)$. Em relação ao nível socioeconômico, a maior parte das crianças com TPAC $(47,83 \%)$ estão no nível médio e, das crianças sem transtorno, $50 \%$ estão no nível mais baixo (Tabela 1 ).

Ao analisar, através do teste de Mann-Whitney, as médias dos resultados de cada teste da bateria diagnóstica mínima de processamento auditivo central entre os grupos estudados, observou-se que não houve diferença estatisticamente significante entre os resultados da orelha esquerda do teste Fala Filtrada $(\mathrm{p}=0,054)$ e da orelha direita do Teste Dicótico de Dígitos $(p=0,082)$ (Tabela 2). Nos demais testes e orelhas o G1 tinha desempenho estatisticamente diferente do G2 nos testes da bateria do PAC.

Constatou-se ao analisar o percentual de crianças do G1, isto é, do grupo com TPAC que apresentaram alterações em cada teste e orelha, que o teste de fusão binaural seguido do teste de fala filtrada em ambas as orelhas foram os testes com menor percentual de crianças do G1 com desempenho alterado. Ao contrário do teste dicótico de dígitos, de ordenação e resolução temporal (Tabela 3). 


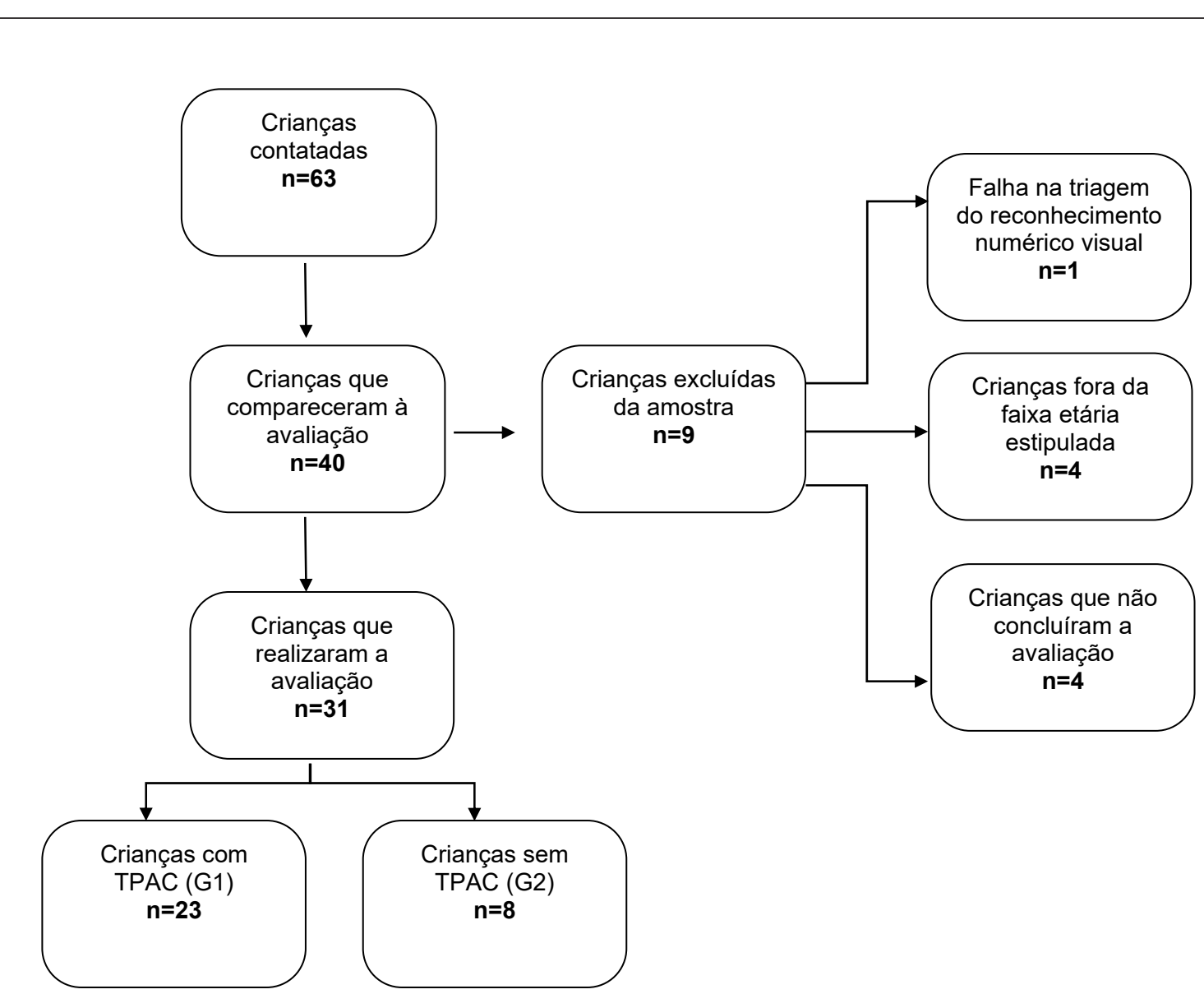

Figura 1. Fluxograma do processo de constituição da amostra do estudo.

Tabela 1. Dados sócio-demográficos da amostra estudada por grupo

\begin{tabular}{lccc}
\hline & $\begin{array}{c}\mathbf{G 1} \\
(\mathbf{n = 2 3})\end{array}$ & $\begin{array}{c}\mathbf{G 2} \\
(\mathbf{n = 8})\end{array}$ & $\begin{array}{c}\text { Total } \\
(\mathbf{n = 3 1})\end{array}$ \\
\hline Idade & & & \\
Média $\pm \mathrm{dp}$ & $10,08 \pm 1,12$ & $10,37 \pm 0,92$ & $10,16 \pm 1,07$ \\
Mínimo-máximo & $8-12$ & $9-12$ & $8-12$ \\
\hline Sexo & & & \\
Masculino & $13(56,52 \%)$ & $7(87,5 \%)$ & $20(64,52 \%)$ \\
Feminino & $10(43,48 \%)$ & $1(12,5 \%)$ & $11(35,48 \%)$ \\
\hline Nível sócio-econômico & $7(21,74 \%)$ & $4(50 \%)$ & $11(35,48 \%)$ \\
Baixo & $11(47,83 \%)$ & $1(12,5 \%)$ & $12(38,71 \%)$ \\
Médio & $5(30,43 \%)$ & $3(37,5 \%)$ & $8(25,81 \%)$ \\
Alto & & & \\
\hline
\end{tabular}

Legenda: $\mathrm{dp}=$ desvio-padrão; $\mathrm{n}=$ número de sujeito; $\%=$ porcentagem 
Tabela 2. Estatística descritiva para caracterização do desempenho da amostra estudada nos testes de processamento auditivo central por grupo

\begin{tabular}{|c|c|c|c|c|}
\hline & & G1 & G2 & p* \\
\hline \multirow{4}{*}{ FF OD } & Média $\pm d p$ & $69,39 \pm 11,02$ & $80 \pm 9,07$ & \multirow{4}{*}{0,016} \\
\hline & Q1 & 64 & 77 & \\
\hline & Mediana & 72 & 84 & \\
\hline & Q3 & 76 & 85 & \\
\hline \multirow{4}{*}{ FF OE } & Média $\pm d p$ & $68,78 \pm 12,25$ & $77,5 \pm 9,30$ & \multirow{4}{*}{0,054} \\
\hline & Q1 & 61 & 72 & \\
\hline & Mediana & 68 & 80 & \\
\hline & Q3 & 76 & 82 & \\
\hline \multirow{4}{*}{ FB OD } & Média $\pm d p$ & $84,69 \pm 7,49$ & $93 \pm 4,14$ & \multirow{4}{*}{0,007} \\
\hline & Q1 & 78 & 91 & \\
\hline & Mediana & 84 & 92 & \\
\hline & Q3 & 92 & 96 & \\
\hline \multirow{4}{*}{ FB OE } & Média $\pm d p$ & $83,91 \pm 6,88$ & $92,5 \pm 3,96$ & \multirow{4}{*}{0,001} \\
\hline & Q1 & 80 & 92 & \\
\hline & Mediana & 84 & 92 & \\
\hline & Q3 & 88 & 96 & \\
\hline \multirow{4}{*}{ DDI OD } & Média $\pm d p$ & $88,69 \pm 11,42$ & $95 \pm 6,05$ & \multirow{4}{*}{0,082} \\
\hline & Q1 & 86,25 & 95 & \\
\hline & Mediana & 90 & 98,75 & \\
\hline & Q3 & 96,25 & 100 & \\
\hline \multirow{4}{*}{ DDI OE } & Média $\pm d p$ & $80,65 \pm 10,79$ & $90,93 \pm 13,41$ & \multirow{4}{*}{0,048} \\
\hline & Q1 & 75 & 83,12 & \\
\hline & Mediana & 87,5 & 97,5 & \\
\hline & Q3 & 90 & 100 & \\
\hline \multirow{4}{*}{ TPF } & Média $\pm d p$ & $68,10 \pm 18,33$ & $91,66 \pm 0$ & \multirow{4}{*}{0,000} \\
\hline & Q1 & 56,66 & 90 & \\
\hline & Mediana & 70 & 90 & \\
\hline & Q3 & 83,31 & 94,14 & \\
\hline \multirow{4}{*}{ GIN OD } & Média $\pm d p$ & $7,95 \pm 3,41$ & $5,25 \pm 0,70$ & \multirow{4}{*}{0,024} \\
\hline & Q1 & 6 & 5 & \\
\hline & Mediana & 8 & 5 & \\
\hline & Q3 & 9,5 & 6 & \\
\hline \multirow{4}{*}{ GIN OE } & Média $\pm d p$ & $7,72 \pm 2,41$ & $5,75 \pm 1,03$ & \multirow{4}{*}{0,024} \\
\hline & Q1 & 6 & 5 & \\
\hline & Mediana & 8 & 5,5 & \\
\hline & Q3 & 8 & 6 & \\
\hline
\end{tabular}

Legenda: *Teste Mann-Whitney; FF=teste fala filtrada; $O D=$ orelha direita; $O E=$ orelha esquerda; $F B=$ teste fusão binaural; $D D I=$ teste dicótico de dígitos (tarefa de integração); TPF=teste padrão de frequência; GIN=teste gaps-in-noise; dp=desvio-padrão; Q1=primeiro quartil; Q3=terceiro quartil; $* * \mathrm{p}<0,05$ 
Tabela 3. Percentual de crianças do $G 1(n=23)$ com alteração em cada teste da avaliação do PAC.

\begin{tabular}{cccc}
\hline & Alterado & $\mathbf{N}(\%)$ & $\begin{array}{c}\text { Normal } \\
\mathbf{N}(\%)\end{array}$ \\
\hline \multirow{2}{*}{ FF } & OD & $3(13,05)$ & $20(86,95)$ \\
& OE & $4(17,40)$ & $19(82,60)$ \\
FB & OD & $1(4,35)$ & $22(95,65)$ \\
& OE & $1(4,34)$ & $22(95,65)$ \\
DDI & OD & $15(65,22)$ & $8(34,78)$ \\
TPF & OE & $20(86,95)$ & $3(13,05)$ \\
& & $14(60,87)$ & $10(39,13)$ \\
GIN & OD & $13(56,52)$ & $8(34,48)$ \\
& OE & $15(65,22)$ & $10(48)$ \\
\hline
\end{tabular}

Legenda: $\mathrm{N}=$ número de crianças; $\mathrm{FF}=$ teste fala filtrada; $\mathrm{OD}=$ orelha direita; $\mathrm{OE}=$ orelha esquerda; $\mathrm{FB}=$ teste fusão binaural; $\mathrm{DDI}=$ teste dicótico de dígitos (tarefa de integração); TPF=teste padrão de frequência; GIN=teste gaps-in-noise.

Na Tabela 4 pode ser observado que também não houve diferença estatisticamente significante entre o G1 e G2 no comparativo das médias de relação sinal-ruído final $(\mathrm{p}=0,227)$ no TDR.

Tabela 4. Estatística descritiva e inferencial do resultado da média do TDR (S/R final) por grupo estudado.

\begin{tabular}{cccc}
\hline & G1 & G2 & p* \\
\hline Média $\pm d p$ & $-7,29 \pm 4,76$ & $-8,42 \pm 2,93$ & \\
Q1 & $-10,40$ & $-9,80$ & 0,227 \\
Mediana & $-9,20$ & $-9,10$ & \\
Q3 & $-6,00$ & $-7,75$ & \\
\hline
\end{tabular}

Legenda: *Teste Mann-Whitney; dp=desvio-padrão; Q1=primeiro quartil; Q3=terceiro quartil; **p $<0,05$

Ao analisar a correlação entre cada teste da avaliação de PAC com os resultados da média de relação sinal/ruído do TDR pode-se observar que não houve correlação, ou ela foi negativa (Tabela 5).

Tabela 5. Análise de Correlação de Spearman entre a média de relação sinal/ruído final e o desempenho dos sujeitos do G1 em cada teste que compõe a bateria mínima para avaliação comportamental do processamento auditivo central.

\begin{tabular}{cccccccccc}
\hline & FF OD & FF OE & FB OD & FB OE & DDI OD & DDI OE & TPF & GIN OD & GIN OE \\
\hline $\begin{array}{c}\text { TDR } \\
\text { (S/R final) }\end{array}$ & 0,109 & $-0,096$ & $-0,368$ & $-0,608$ & $-0,504$ & $-0,254$ & $-0,108$ & 0,203 & 0,152 \\
P* $^{*}$ & 0,619 & 0,665 & 0,084 & 0,002 & 0,014 & 0,241 & 0,622 & 0,353 & 0,488 \\
$\mathrm{R}^{2}$ & 0.056 & 0,025 & 0,139 & 0,354 & 0,432 & 3,821 & 4,951 & 0,002 & 0,002 \\
\hline
\end{tabular}

Legenda: *Coeficiente de Correlação de Spearman; FF=teste fala filtrada; $O D=$ orelha direita; OE=orelha esquerda; $F B=$ teste fusão binaural; DDI=teste dicótico de dígitos (tarefa de integração); TPF=teste padrão de frequência; GIN=teste gaps-in-noise; TDR=teste de dígitos no ruído; $\mathrm{S} / \mathrm{R}=$ relação sinal/ruído; $* * \mathrm{p}<0,05$

\section{Discussão}

Neste estudo evidenciamos que o desempenho das crianças com TPAC é similar ao de crianças sem TPAC no teste de dígitos no ruído em Português
Brasileiro, em virtude da ausência de significância estatística entre os dois grupos.

O sinal acústico e a tarefa proposta pelo TDR envolvem o reconhecimento da fala no ruído. Crianças com audição normal a partir dos quatro anos de idade conseguem realizar o teste com a 
tarefa de reconhecimento de fala no ruído para avaliar essa habilidade, que depende da capacidade da criança em dissociar o sinal de fala do ruído de fundo, de se beneficiar das flutuações acústicas do ruído e das pistas binaurais ${ }^{3}$. Desse modo, buscou-se verificar a aplicabilidade do TDR como uma ferramenta de triagem do TPAC, partindo inicialmente do comparativo entre um grupo de crianças com esta alteração e outro grupo que não apresentasse TPAC.

Vale salientar que algumas controvérsias permeiam a definição e o diagnóstico do TPAC ${ }^{24-28}$. Não há como assegurar que se trata de um déficit somente com características auditivas ${ }^{28}$. As evidências científicas fortalecem a premissa de que diferentes transtornos com modulação top-down como Distúrbio Específico de Linguagem, Transtorno do Déficit de Atenção e Hiperatividade, Dislexia do Desenvolvimento, Dificuldade de Aprendizagem e o TPAC têm manifestações semelhantes, e até coincidentes, quanto a questões como inteligência, memória, atenção e linguagem ${ }^{27}$.

Há ainda o conceito de que o TPAC e todas as suas manifestações sintomáticas vão além de uma desordem, e se constituem como um importante marcador da Síndrome Neurodesenvolvimental, que associa diversos marcadores nos aspectos auditivos, de fala, atenção, memória e dificuldades comportamentais. A depender da severidade e do predomínio de um ou mais marcadores, a criança manifesta um perfil de desenvolvimento singular com desdobramentos modulados através da composição genética, do ambiente a que a criança está exposta, da idade e das demandas acadêmicas ${ }^{24}$. Por um lado, acredita-se que crianças com um transtorno na função auditiva central não podem ser diagnosticadas apenas com uma combinação de testes, que parecem não seguir a mesma lógica das características apresentadas ${ }^{25,29}$.

Em contrapartida, as ferramentas de avaliação diagnóstica disponíveis, através de uma bateria mínima de testes, se mostram sensíveis para detectar a disfunção de processamento auditivo central em regiões corticais específicas, a sua natureza de acordo com os pontos fortes e as inabilidades identificadas, bem como quantificar e qualificar as dificuldades auditivas, associadas a alterações comunicativas, de aprendizagem e demais comorbidades ${ }^{26,30}$. Sendo assim, foi utilizada para esse estudo uma bateria comportamental mínima composta pelos testes Fala Filtrada, que avalia a habilidade de fechamento auditivo; Fusão Binaural, para avaliar a habilidade de síntese auditiva; Dicótico de Dígitos, avaliando a integração binaural; Teste Padrão de Frequência, responsável por avaliar a habilidade de ordenação temporal e o teste Gaps-in-noise (GIN) para avaliar a resolução temporal ${ }^{18,19,31}$.

A ausência de correlação entre os testes de fala filtrada, fusão binaural OD, dicótico de dígitos $\mathrm{OE}$, TPF e GIN OE e OE com os resultados do TDR nas crianças com TPAC evidenciam que o TDR não distingue crianças com TPAC das que não tem TPAC. Já a correlação negativa entre o teste de fusão binaural $\mathrm{OE}$ e o dicótico de dígitos $\mathrm{OD}$ e os resultados do TDR demonstram que quanto maior o desempenho nestes testes menor no TDR, pois as variáveis se correlacionam negativamente. Portanto, mesmo o teste de fala filtrada que avalia a habilidade de fechamento auditivo e, em teoria, poderia ter uma correlação maior com o reconhecimento de fala no ruído solicitado no TDR às crianças não apresentou diferença entre os grupos estudados.

Este resultado pode ter sido influenciado pela baixa ocorrência na amostra de 23 crianças (46 orelhas) de resultados abaixo do esperado no teste de fala filtrada. Os testes monoaurais de baixa-redundância são os testes que envolvem a diminuição da redundância extrínseca do sinal de fala, através de distorção do estímulo com filtros passa-baixo, compressão do tempo, ruído competitivo e reverberação, capazes de avaliar a habilidade de fechamento auditivo, que consiste na capacidade de reconhecer a informação auditiva quando não há clareza ${ }^{2,30}$. Contudo, essa habilidade não é a única dentro do escopo para detectar o TPAC ${ }^{2}$, o que pode configurar uma importante influência na ausência de achados clínicos ao diferenciar o G1 do G2 e o próprio comparativo dos resultados do TDR. Aponta-se, ainda, a importância de testes para triagem de PAC que envolvam, principalmente, as habilidades de escuta dicótica, localização, lateralização e demais interações binaurais por evidenciarem maior sensibilidade na detecção do TPAC e demais alterações no Sistema Nervoso Auditivo Central ${ }^{2}$.

Entre as limitações enfrentadas durante as etapas de desenvolvimento deste estudo, é possível elencar a dificuldade na coleta de dados clínicos e ampliação do $n$ amostral do grupo de crianças sem TPAC, a pouca adesão dos pais ou responsáveis em comparecer ou retornar para finalização 
dos procedimentos clínicos e, por fim, a falta de disponibilidade de horários dos pais para os atendimentos no Ambulatório.

A falta de equiparação do $n$ amostral nos grupos avaliados repercute nos resultados encontrados e leva a hipotetizar que a ausência de diferença estatisticamente significante ao comparar os resultados do teste FF na orelha esquerda poderia ser elucidada com grupos nivelados, visto que o valor de $p$ está muito próximo da significância. Seria ainda necessário correlacionar o TDR com testes comportamentais que avaliam o reconhecimento de fala no ruído com propostas de tarefas semelhantes. É o caso, portanto, do HINT ${ }^{9,10}$ e do subteste Fala no Ruído do SCAN ${ }^{5,6}$.

No entanto, destaca-se que na maior parte dos testes e orelhas avaliadas com os procedimentos de avaliação do PAC, o G1 apresentou desempenhos inferiores e estatisticamente diferentes do G2, o que caracteriza que, de fato, são sujeitos com espectros diferentes nas habilidades auditivas, sendo que no TDR apresentaram resultados semelhantes.

Desta forma, são necessários novos estudos com $n$ amostral mais elevado para vir a confirmar os achados presentes, especialmente no grupo sem alteração, com o intuito de viabilizar a análise de acurácia deste instrumento. Caso se confirmem, haverá evidência ao fato que o TDR de forma diótica como apresentado neste estudo poderá não ser um teste válido para ser utilizado com crianças com TPAC. Sugere-se, também, estudar a apresentação dicótica do TDR em futuros estudos, pois talvez possa ser potencializado para avaliar o Sistema Nervoso Auditivo Central.

\section{Conclusão}

O desempenho das crianças com TPAC é similar ao de crianças sem TPAC no teste de dígitos no ruído em Português Brasileiro.

\section{Agradecimentos}

Agradecemos especialmente a todas as crianças e aos familiares que se disponibilizaram a participar deste estudo. Também agradecemos aos colegas da base de pesquisa pelas contribuições durante o desenvolvimento desta pesquisa. Além dos pesquisadores De Wet Swanepoel, Cas Smits, Deborah Vivianne Ferrari e Hannalice G. Cavalcanti que contribuíram no desenvolvimento do teste de dígitos no ruído na versão em Português Brasileiro.

\section{Referências bibliográficas}

1. American Speech-Language-Hearing Association (2005) (Central) auditory processing disorders: Technical Report. 2005. [citado em 15 mai 19]. Available at https://find.asha. org/asha $\# \mathrm{q}=\% 40$ keywords $\% 3 \mathrm{D}$ Auditory $\% 2 \mathrm{~B}$ Processing \&sort=relevancy

2. AAA American Academy of Audiology Clinical Practice Guidelines: Diagnosis, Treatment and Management of Children and Adults with Central Auditory Processing Disorder. 2010 [citado em 3 mai 19]. Avaliableat: http://www.audiology.org/ publications-resources/document-library/central-auditoryprocessing-disorder

3. Smits C, Goverts ST, Festen JM. The digits-in-noise test: Assessing auditory speech recognition abilities in noise. J AcoustSocAm. 2013; 133(3): 1693-706.

4. Pereira LD, Schochat E. Testes Auditivos Comportamentais para Avaliação do Processamento Auditivo Central. Barueri (SP): Pró-Fono. 2011.

5. Keith RW. Development and standardization of SCAN-C Test for Auditory Processing Disorders in Children. J AmAcadAudiol. 2000; 11(8): 438-45.

6. Zaidan E. Elaboração de um material de triagem do processamento auditivo central para crianças de 6 a 11 anos. São Paulo: Faculdade de Medicina da Universidade de São Paulo, 2001. Trabalho de Conclusão de Curso (dissertação).

7. Yathiraj A, Maggu AR. Validation of the Screening Test for Auditory Processing (STAP) on school-aged children. Int J Pediatr Otorhinolaryngol. 2014; 78(3): 479-88.

8. Nickisch A, Heuckmann C, Burger T, Massinger C. Münchner Auditiver Screening test für Verarbeitungs-und Wahrnehmungs störungen (MAUS). Laryngo-Rhino-Otol. 2006; 85(4): 253-9.

9. Nilsson M, Soli SD, Sullivan JA. Development of the Hearing in Noise Test for the measurement of speech reception thresholds in quiet and in noise. J AcoustSocAm. 1994; 95(2): 1085-99.

10. Bevilacqua MC, Banhara MR, Costa EA, Vignoly AB, Alvarenga KF. The Brazilian Portuguese hearing in noise test. Int J Audiol. 2008; 47(6): 364-5.

11. Smits C, Kapteyn TS, Houtgast T. Development and validation of an automatic speech-in-noise screening test by telephone. Int J Audiol. 2004; 43(1): 15-28.

12. Smits C, Merkus P, Houtgast T. How we do it: The Dutch functional hearing screening tests by telephone and internet. ClinOtolaryngol. 2006; 31(5): 436-40.

13. Potgieter JM, Swanepoel DW, Myburgh HC, Hopper TC, Smits C. Development and validation of a smartphone-based digits-in-noise hearing test in South African English. Int J Audiol. 2016; 55(7): 405-11.

14. Potgieter JM, Swanepoel DW, Smits C. Evaluating a smartphone digits-in-noise test as part of the audiometric test battery. SAJCD. 2018; 65(1):1-6.

15. Desousa KC,Swanepoel DW, Moore DR, Myburgh HC, Smits C. Improving Sensitivity of the Digits-In-Noise Test Using Antiphasic Stimuli. EarHear, 2019; 41(2): 442-50. http:// doi.org/10.1097/AUD.0000000000000775.

16. Koopmans WJA, Goverts ST, Smits C. Speech recognition abilities in normal-hearing children 4 to 12 years of age in stationary and interrupted noise. EarHear. 2018; 39(6): 1091103. 
17. Andrade M. Avaliação do Teste de Dígitos no Ruído em português brasileiro em uma população com audição normal Metodologia nas Humanidades. João Pessoa: Universidade Federal da Paraíba, 2019. Trabalho de Conclusão do Programa Associado de Pós-Graduação em Fonoaudiologia entre UFRN e UFPB.

18. Melo IMM. Acurácia de testes de triagens auditivas via smartphones para a identificação da deficiência auditiva. Natal: Universidade Federal do Rio Grande do Norte, 2019. Trabalho de Conclusão do Programa Associado de Pós-Graduação em Fonoaudiologia entre UFRN e UFPB.

19. Potgieter JM, Swanepoel DW, Smits C. Evaluating a smartphone digits-in-noise-test as part of the audiometric test battery. South African Journal of Communication Disorders. 2018; 65(1); a574. Doi.org/10.4102/sajcd.v65i1.574.

20. Silman S, Silverman CA. Basic Audiologic Testing. In: Silman S, Silverman CA. Auditory diagnosis: principles and applications. San Diego: Singular Publishing Group; 1997; 44-52.

21. Carvallo RMM, Sanches SGG. Medidas de Imitância Acústica. In: Boécha EM, Menezes PL, Couto CM, Frizzo ACF, Scharlach RC, Anastasio ART, organizadores. Tratado de Audiologia. $2^{\mathrm{a}}$ ed. Rio de Janeiro: Guanabara Koogan; 2015.

22. ABEP, Associação Brasileira de Empresas de Pesquisa. Critério Brasil. 2018 [citado em 31 jul 20]. Disponível em: http://www.abep.org/criterio-brasil

23. AUDITEC. GIN and Pitch Pattern Sequence Test. Auditec St. Louis. 2017 [citado em 12 abr 19]. Avaliableat: http://www. auditec.com/

24. 24 - 22. Moore DR, Hunter LL. Auditory processing disorder (APD) in children: A marker of neuro developmental syndrome. Hearing Balance Communication. 2013; 11(3): 160-7.

25. Moore DR. Guest Editorial: Auditory processing disorder. Ear Hear. 2018; 39(4): 617-20

26. Iliadou V, Chermak GD, Bamiou DE, Rawool VW, Ptok M, Purdy S, et al. Letter to the editor: An affront to scientific inquiry re: Moore, D. R. (2018) Editorial: Auditory processing disorder, EarHear, 39, 617-620. EarHear. 2018; 39(6): 1236-42.

27. de Wit E, van Dijk P, Hanekamp S, Visser-Bochane MI, Steenbergen B, van der Schans CP, et al. Same or Different: The Overlap Between Children With Auditory Processing Disorders and Children With Other Developmental Disorders: A Systematic Review. EarHear. 2018; 39(1): 1-19.

28. Neijenhuis K, Campbell NG, Cromb M, Luinge MR, Moore DR, Rosen S, et al. An Evidence-Based Perspective on "Misconceptions" Regarding Pediatric Auditory Processing Disorder. FrontNeurol. 2019; 10(March): 1-4.

29. Emanuel DC, Ficca KN, Korczak P. Survey of the Diagnosis and Management of Auditory Processing Disorder. Am J Audiol. 2011; 20(1): 48-60

30. Bellis TJ, Ferre JM. Multidimensional Approach to the Differential Diagnosis of Central Auditory Processing Disorders in Children. J AmAcadAudiol. 1999; 10(6): 319-28.

31. Musiek FE, Shinn JB, Jirsa R, Bamiou DE, Baran JA, Zaida E. GIN (Gaps-In-Noise) test performance in subjects with confirmed central auditory nervous system involvement. EarHear. 2005; 26(6): 608-18. 